\title{
Continuing Cancer Treatment in a "COVID HOTSPOT" in India: Are We Overestimating the Risks?
}

\begin{abstract}
Context: We describe the treatment of cancer patients carried out in a Government of India-designated, dedicated coronavirus disease (COVID) hospital (DCH) in a COVID hotspot in India. Aims: The aim was to study the change and delay in the management of cancer patients during the pandemic and its complications. Settings and Design: This was an observational cohort study conducted at a tertiary care center, which was also a DCH. Subjects and Methods: Cancer patients receiving cancer surgery, chemotherapy, and radiotherapy in our $\mathrm{DCH}$, during the lockdown, were studied. Results: A total of 864 patients received treatment for cancer in our hospital during the period of March 20, 2020 - May 31, 2020. There were no COVID-related complications. The treatment of $109 / 864$ patients $(12.61 \%)$ was delayed due to the pandemic and lockdown situation and the treatment plan was changed for $84 / 864(9.72 \%)$ patients. There were 21 deaths in these 864 patients $(2.43 \%)$, but only two deaths were COVID related. Symptomatic patients were tested for COVID, and 3/864 patients $(0.34 \%)$ were detected to be COVID positive. Conclusions: We successfully delivered cancer treatment to patients in our DCH. The percentage of adverse effects, symptomatic COVID infection, and related mortality has been very low in our study. Cancer care can be continued with due diligence even during this pandemic.
\end{abstract}

Keywords: Cancer surgery, cancer treatment, chemotherapy, COVID-19 pandemic, radiotherapy

\section{Introduction}

The coronavirus disease 2019 (COVID-19) pandemic has deemed it necessary for clinicians to view patient treatment guidelines in the context of the danger of hospital-acquired COVID infection. Elective surgeries and procedures have been postponed and only emergency and lifesaving procedures are carried out.

Cancer treatment places different challenges in front of oncologists because delay in therapy can result in progression of cancer with worsening of outcome. ${ }^{[1]}$

The first patient of COVID in Pune, Maharashtra, India, was detected on March 9, 2020. Thereafter, the number of patients in Pune district and Maharashtra state kept on increasing exponentially, and hence, Pune has been labeled as a hotspot for COVID infection [Figures 1a-c]. The Government of India announced a nationwide lockdown on March 24, 2020, which was extended in different phases. At the time of writing this article, India was

This is an open access journal, and articles are distributed under the terms of the Creative Commons Attribution-NonCommercial-ShareAlike 4.0 License, which allows others to remix, tweak, and build upon the work non-commercially, as long as appropriate credit is given and the new creations are licensed under the identical terms.

For reprints contact: WKHLRPMedknow_reprints@wolterskluwer.com in its $5^{\text {th }}$ phase of lockdown, from June 1 , to June 30 . We present a cohort study of cancer patients treated at our tertiary care designated COVID hospital.

\section{Aims and objectives}

Our aim has been to study the change and delay in the management of cancer patients in this pandemic and lockdown situation. We have studied the comorbidity profile and complications in these patients and evaluated symptomatic patients for COVID infection in the study period and the follow-up period till June 15.

\section{Subjects and Methods}

This was a cohort study in which we evaluated patients receiving cancer treatment in our hospital during the period of March 20, 2020 - May 31, 2020. They were further followed up till June 15, 2020, considering the incubation period for COVID-19 infection. The institutional ethics committee approved this study.

All newly registered patients who underwent cancer surgery, chemotherapy,

How to cite this article: Parasnis AS, Kelkar DS, Khaladkar BS, Kurlekar UA, Deshmukh CD, Pingle $\mathrm{S}$, et al. Continuing cancer treatment in a "COVID HOTSPOT" in India: Are we overestimating the risks? Indian J Med Paediatr Oncol 2020;41:634-9.

\section{Amit Surendra \\ Parasnis, \\ Dhananjay S Kelkar, Bhagyashree Suparn Khaladkar, Utkrant A Kurlekar, Chetan D Deshmukh, Sonali Pingle, Sachin S Hingmire, Padmaj S Kulkarni, Shailesh S Shende, Girish K Phadke, Mahesh B Sambhus, Sameer R Melinkeri, Shailesh A \\ Kanvinde}

Department of Oncosurgery, Medical Oncology and Radiation Oncology, Deenanath Mangeshkar Hospital and Research Centre, Erandwane, Pune, Maharashtra, India

Submitted: 09-Jul-2020 Revised: 24-Aug-2020

Accepted: 24-Aug-2020

Published: 29-Oct-2020

\section{Address for correspondence:} Dr. Amit Surendra Parasnis, Department of Oncosurgery, Medical Oncology and Radiation Oncology,

Deenanath Mangeshkar

Hospital and Research Centre,

Erandwane, Off Karve Road,

Pune - 411 004,

Maharashtra, India.

E-mail: amitparasnis@gmail. com

Access this article online Website: www.ijmpo.org DOI: 10.4103/ijmpo.ijmpo_327_20 Quick Response Code:

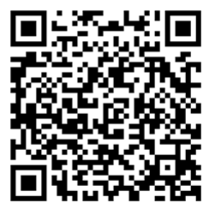


radiotherapy, or palliative care in our hospital during the study period were included in the study. Those patients who were already receiving treatment for cancer were also included. Cancer patients who were only on supportive care treatment were excluded from the study.

Consent was obtained from the patients included in the study. Information gathered for this study included patient demographics, comorbidity profile, delay in starting treatment, complications, and COVID-19 infection and related morbidity in patients. Delay was defined as any delay in initiating planned treatment of $>7$ days.

The required data were obtained from the patients, treating doctors, and electronic medical records of the hospital.

Microsoft Excel Version 22019 16.0.6742.2048 (Microsoft Corporation, Redmond, Washington, USA) and IBM SPSS Statistics for Windows version V26 (IBM Corp., Armonk, N.Y., USA) were used for data recording and analysis. Chi-square test was used to rule out any difference between

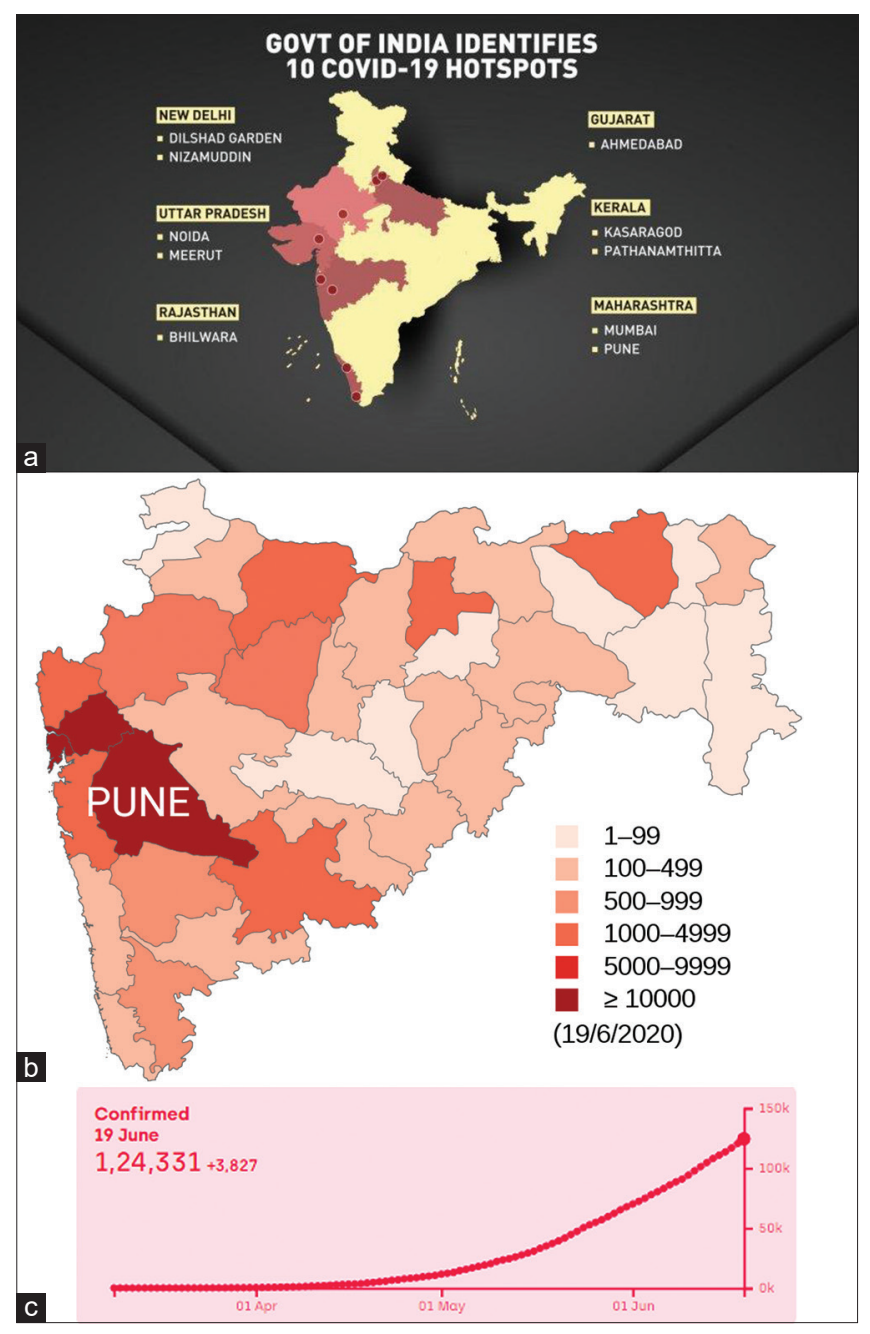

Figure 1: (a) COVID-19 hotspots in India (Source: Wionews.com), (b) prevalence of COVID-19 in the state of Maharashtra, with Pune being a hotspot (Source: Wikipedia), (c) rising COVID-19 patients in Pune (Source: COVID-19 India.org) the mortality during the study period and the mortality during the preceding 2 months.

\section{Results}

A total of 940 patients underwent cancer care at our hospital. Out of these, 864 patients who underwent surgery, chemotherapy, or radiation were included for analysis [Figure 2].

Patient characteristics regarding demographics and comorbidities are mentioned in Table 1. The follow-up period was 15-78 days, with a median of 37 days.

One hundred and ten patients underwent 122 surgeries, out of which 102 were major and 20 were minor surgeries. The median hospital stay was 4 days, with a range of 1-22 days. None of the surgical complications seen fitted into the profile of COVID-19 infection.

Six hundred and fifty-eight patients received chemotherapy [Table 2], and a total of 1888 cycles were administered during the study period. Bone marrow transplant was done in 11 patients. Two hundred and five patients received radiotherapy [Table 3] during the study period, and 3498 fractions were administered. Information regarding delay in initiating treatment and complications during cancer therapy is given in Table 1 and Figure 3a-c.

Surgical treatment decision had to be modified only in $1 / 110(0.9 \%)$ patient of rectal cancer. She preferred surgery to neoadjuvant chemoradiotherapy because it required a greater number of hospital visits. Similarly, in $46 / 658$ patients (6.99\%) undergoing chemotherapy [Table 4] and in 37/205 (18.04\%) patients

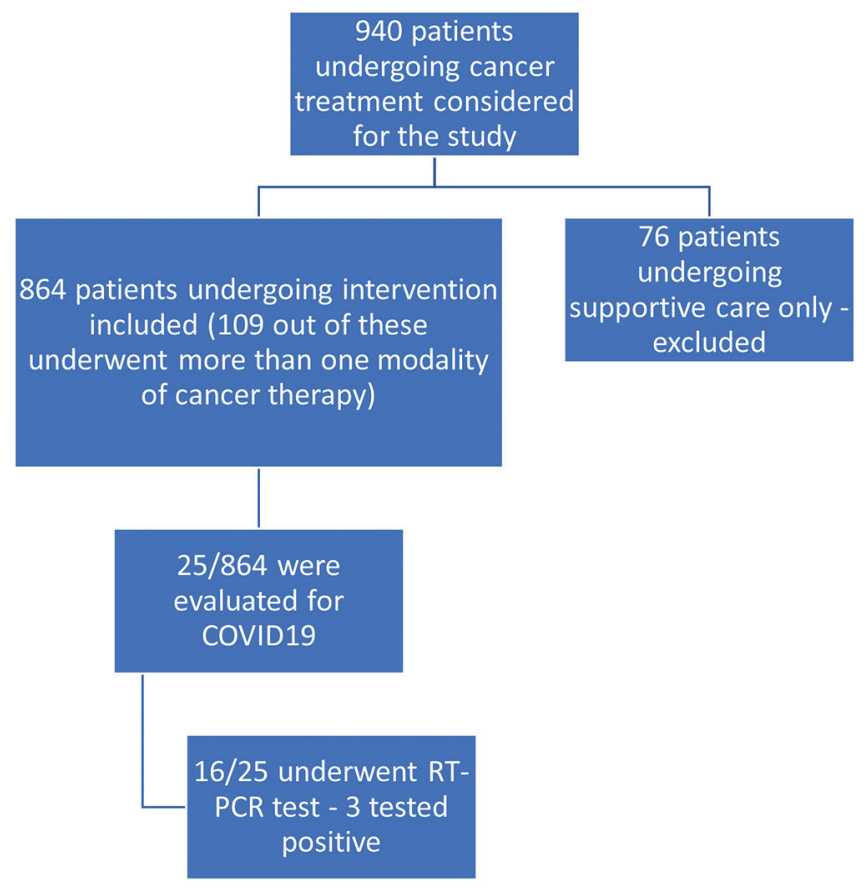

Figure 2: Participants' flowchart 


\begin{tabular}{|c|c|}
\hline \multicolumn{2}{|c|}{ Table 1: Demographics and other details } \\
\hline Variable & $\begin{array}{c}\text { Number of } \\
\text { patients }\end{array}$ \\
\hline \multicolumn{2}{|l|}{ Sex } \\
\hline Male & 353 \\
\hline Female & 511 \\
\hline \multicolumn{2}{|l|}{ Age (years) } \\
\hline$<20$ & 65 \\
\hline $21-40$ & 103 \\
\hline $41-60$ & 398 \\
\hline$>60$ & 298 \\
\hline \multicolumn{2}{|l|}{ Comorbidity } \\
\hline Diabetes mellitus & 161 \\
\hline Ischemic heart disease & 34 \\
\hline Hypertension & 71 \\
\hline Bronchial asthma & 11 \\
\hline Obesity & 86 \\
\hline \multicolumn{2}{|l|}{$\begin{array}{l}\text { Delay due to the COVID pandemic ( } n=109 / 864 \text {; } \\
12.61 \%)\end{array}$} \\
\hline Surgery & 11 \\
\hline Chemotherapy & 75 \\
\hline Radiotherapy & 23 \\
\hline \multicolumn{2}{|l|}{$\begin{array}{l}\text { Change in treatment in view of the pandemic } \\
(n=84 / 864 ; 9.72 \%)\end{array}$} \\
\hline Surgery & 1 \\
\hline Chemotherapy & 46 \\
\hline Radiotherapy & 37 \\
\hline \multicolumn{2}{|l|}{ Deaths during the study period $(n=21 / 864 ; 2.43 \%)$} \\
\hline Surgery & 0 \\
\hline Chemotherapy & 16 \\
\hline Radiotherapy & 5 \\
\hline \multicolumn{2}{|l|}{$\begin{array}{l}\text { Hospital visits (each admission/radiation fraction } \\
\text { is counted as one visit) }\end{array}$} \\
\hline Surgery & 122 \\
\hline Chemotherapy & 1888 \\
\hline Radiation & 3498 \\
\hline Total & 5508 \\
\hline $\begin{array}{l}\text { Deaths in patients undergoing cancer treatment } \\
\text { during January-February } 2020(2.24 \%)\end{array}$ & $\begin{array}{c}26 \text { (out of } \\
1157 \text { patients) }\end{array}$ \\
\hline
\end{tabular}

undergoing radiotherapy [Table 5], treatment had to be modified in view of the pandemic situation.

Out of the 25 patients who had symptoms suggestive of COVID, 16 underwent reverse transcription-polymerase chain reaction (RT-PCR) testing, as advised by the COVID treatment team because they fitted into the clinical picture of the disease [Figure 4]. Of the three patients $(3 / 864$, $0.34 \%$ ) who tested positive, one had mild symptoms, while the other two $(2 / 864,0.23 \%)$ patients were suffering from advanced hemato-lymphoid malignancies and succumbed to the infection.

In the 2 months preceding the pandemic, there were 1157 admissions in the oncology department with 26 deaths $(2.24 \%)$. The deaths in the study period are categorized in Figure 5. There were 21 deaths
$(21 / 864,2.43 \%)(P=0.791)$, out of which two were attributed to COVID. There was no statistically significant increase in mortality in the study period. Chi-square test was used to rule out a difference in mortality during the two periods.

\section{Discussion}

In the current COVID pandemic in India, multispecialty hospitals play a huge role in treating COVID patients. These hospitals must also continue treating patients with other ailments during this period. Our hospital is an 800-bedded tertiary care hospital with a fully functional oncology unit.

After the announcement of the nationwide lockdown, an embargo on elective surgical procedures was imposed by the government in light of several reports of hospital-acquired COVID infections in operated patients and reporting of worse outcomes and increased mortality in COVID patients undergoing surgery. ${ }^{[2,3]}$ Consequent to this, a decision to defer elective surgeries, except surgical emergencies, cancer surgeries, and cancer treatment, was taken. This viewpoint from the Indian perspective, where the rise in COVID cases was still slow, was supported by guidelines provided by one of the premier cancer institutes of the country. ${ }^{[4]}$

With a view to separate infectious patients from others, an entire building was designated for COVID patients and another one for non-COVID patients. ${ }^{[5]}$

The following standard operating procedures (SOPs) were laid down to ensure that cancer care delivery continued in the hospital without being affected by the pandemic situation: ${ }^{[5]}$

1. The number of relatives accompanying patients and outpatient visits was restricted

2. The two different buildings had separate outpatient departments, inpatient wards, operating rooms, and radiology services designated for COVID and non-COVID patients

3. Access points to the hospital were limited and they were manned by health-care workers. An effort was made to direct the flow of COVID-positive or suspected positive patients in a specific manner after screening

4. Education of health-care workers, resident doctors, and managers about specific biosafety precautions was carried out by lectures, simulations, and one-to-one meetings

5. Entrance to the chemotherapy day care was restricted, and the health-care workers were educated about social distancing and sanitization

6. Use of specific modified personal protective equipment for managing all surgical patients was made mandatory ${ }^{[5]}$

7. The radiotherapy department couch and headrest were sanitized after each use

8. Appropriate personal protective measures were adopted by the radiotherapy department personnel 


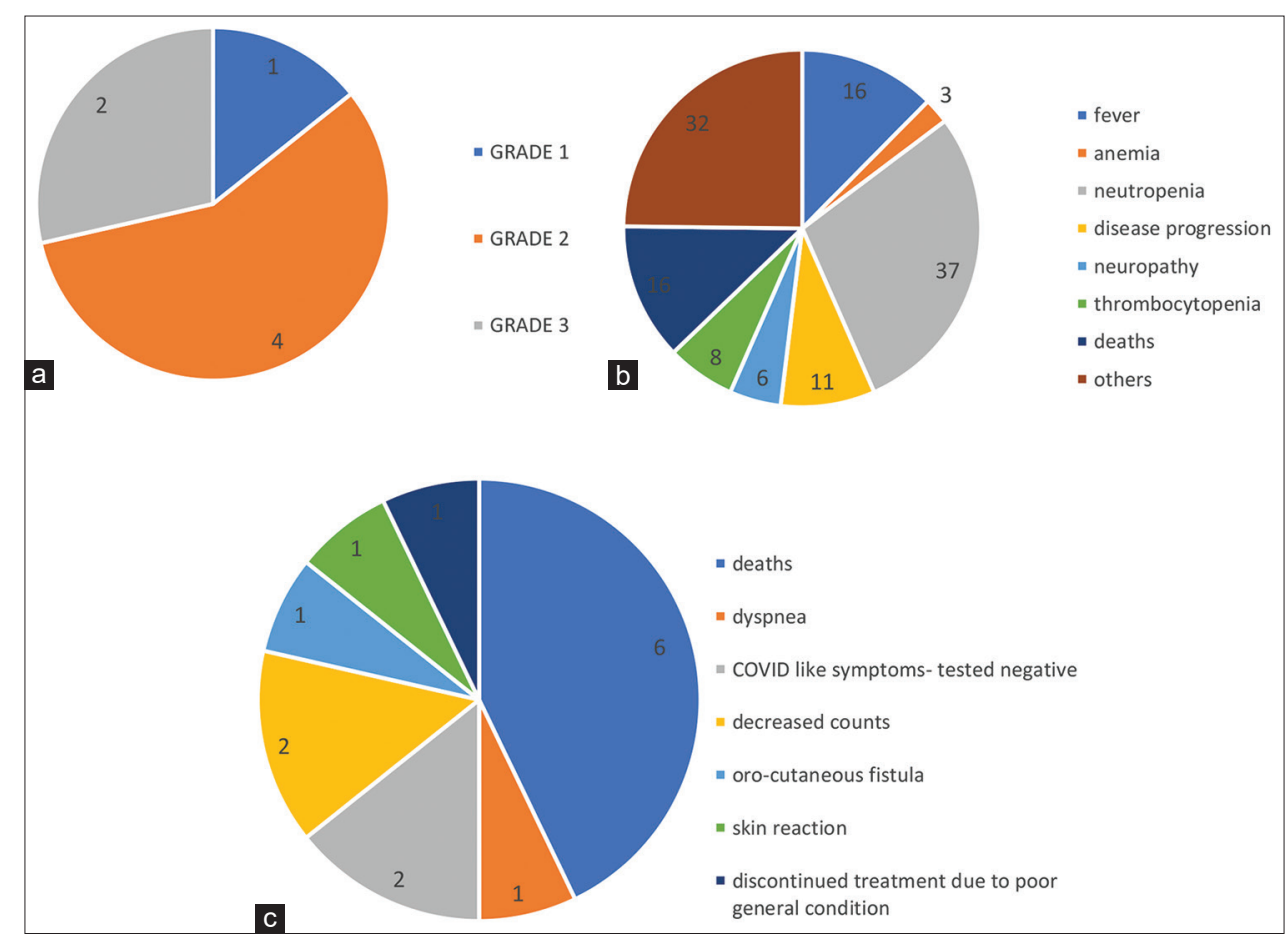

Figure 3: (a) Surgery complications ( $n=7,6.36 \%$ ), (b) chemotherapy complications $(n=129,19.66 \%)$, (c) radiotherapy complications $(n=14,6.82 \%)$

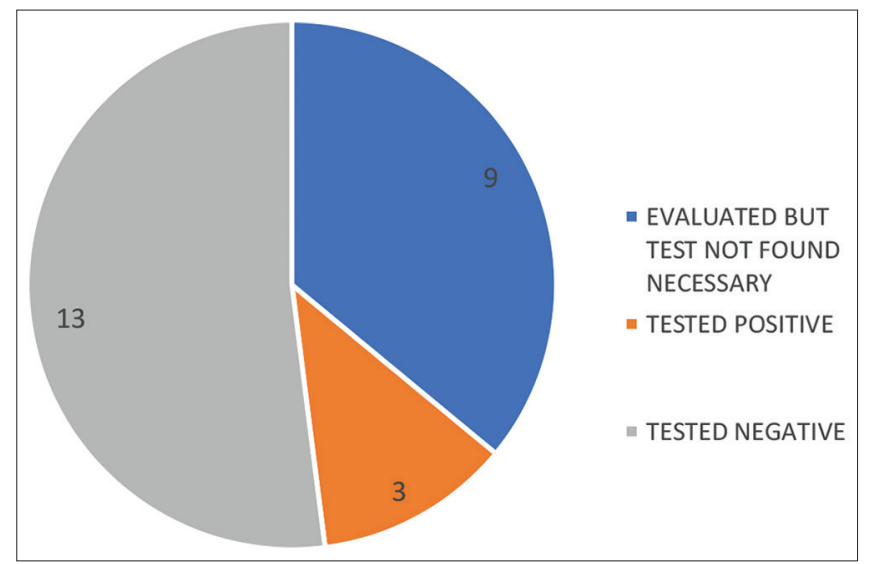

Figure 4: Patients evaluated for COVID ( $n=25,2.89 \%)$

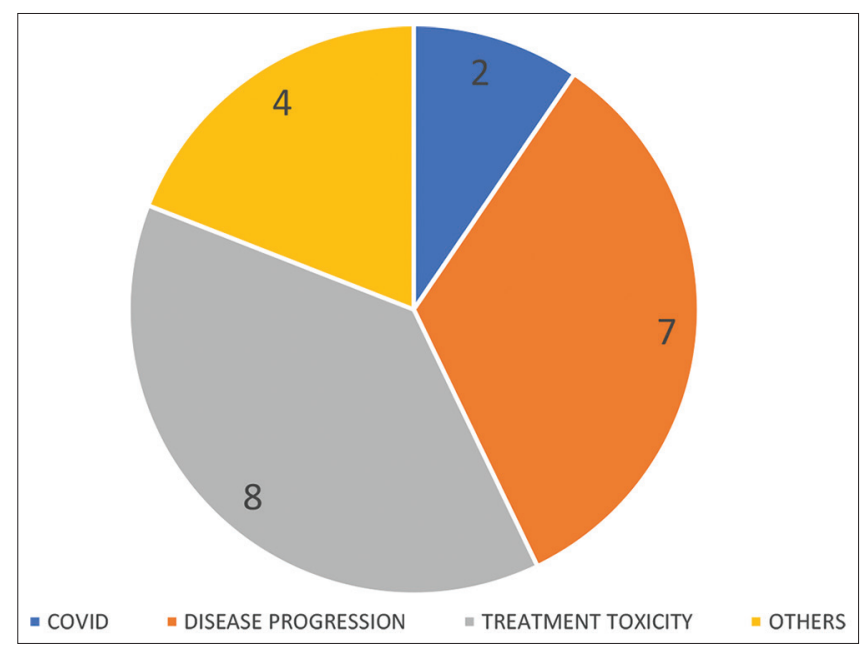

Figure 5: Deaths during the study period $(n=21,2.43 \%)$
9. Appointments for radiotherapy were adequately spaced out to avoid overcrowding

10. Expedited management of surgical patients by liberal use of frozen section analysis instead of paraffin section to minimize the time and visits required for a diagnosis.

Several meetings were held between the medical director and all oncologists. Some changes in the management of common cancers according to recent guidelines issued by various oncology societies were considered. ${ }^{[6]}$ It was unanimously decided that the situation would be reviewed periodically and any increased rate of COVID infection in these cancer patients would warrant a change in strategy.

In accordance with the prevailing Indian Council for Medical Research guidelines, ${ }^{[7]}$ a decision of not doing RT-PCR testing for all patients undergoing cancer treatment was taken. This was contradictory to suggestions by certain authors who recommended preoperative testing for all patients in light of hazardous postoperative complications in COVID-positive patients undergoing surgery. ${ }^{[8]}$

In our series, very few patients required evaluation for COVID infection despite there being multiple hospital visits $(25 / 864,2.893 \%)$. A scrupulous selection of patients and careful peri-operative care has helped in having no COVID-related complications or cross-infections in surgical patients. The incidence of COVID positivity in all treated cancer patients was also very low $(3 / 864,0.34 \%)$ as compared to the other series reported by Shrikhande et al. ${ }^{[9]}$

The incidence of complications in surgery and adverse events in chemotherapy and radiation (150/864, 17.36\%) was comparable to historical controls, and there was no 


\begin{tabular}{|c|c|c|}
\hline \multicolumn{3}{|c|}{ Table 2: Cancer site/type in patients } \\
\hline & Site/type & Number of patients \\
\hline \multirow{11}{*}{$\begin{array}{l}\text { Cancer site/type } \\
\text { in all patients } \\
(n=864)\end{array}$} & Head and neck & 111 \\
\hline & Breast & 236 \\
\hline & Gastrointestinal & 102 \\
\hline & Gynecological & 109 \\
\hline & Urological & 49 \\
\hline & Lung & 41 \\
\hline & Central nervous system & 9 \\
\hline & Soft-tissue sarcomas & 21 \\
\hline & Lymphomas & 87 \\
\hline & Leukemias & 68 \\
\hline & Others & 31 \\
\hline \multirow{7}{*}{$\begin{array}{l}\text { Surgical patients } \\
(n=110)\end{array}$} & Head and neck & 27 \\
\hline & Breast & 31 \\
\hline & Gastrointestinal & 14 \\
\hline & Urological & 15 \\
\hline & Gynecological & 10 \\
\hline & Soft-tissue sarcomas & 5 \\
\hline & Others & 8 \\
\hline
\end{tabular}

\begin{tabular}{llc}
\hline \multicolumn{3}{c}{ Table 3: Intent of treatment } \\
\hline & Intent & Number of patients \\
\hline Chemotherapy $(n=658)$ & Neoadjuvant & 65 \\
& Definitive & 160 \\
& Adjuvant & 168 \\
& Palliative & 222 \\
Radiation $(n=205)$ & Neoadjuvant & 13 \\
& Definitive & 19 \\
& Adjuvant & 105 \\
& Palliative & 68 \\
\hline
\end{tabular}

\begin{tabular}{lc}
\hline \multicolumn{2}{c}{ Table 4: Chemotherapy modifications } \\
\hline Treatment modification in chemotherapy & Number of patients \\
\hline Weekly to 3 weekly & 27 \\
Bi-weekly to 3 weekly & 2 \\
Change of drugs & 15 \\
Shifted to hormonal & 2 \\
Total (\%) & $46 / 658(6.99)$ \\
\hline
\end{tabular}

\begin{tabular}{lc}
\hline \multicolumn{2}{c}{ Table 5: Radiotherapy modifications } \\
\hline Treatment modification in radiotherapy & Number of patients \\
\hline Reduction in number of fractions & 9 \\
Reduction in number of brachytherapy fractions & 8 \\
Hypofractionation & 16 \\
Concurrent chemotherapy omitted & 3 \\
Only brachytherapy & 1 \\
Total $(\%)$ & $37 / 205(18.04)$ \\
\hline
\end{tabular}

unexpected increase in this number due to the impact of pandemic-related events.

Only 109/864 (12.61\%) patients had to suffer a delay for starting their cancer treatments due to the pandemic situation.
This was found in concordance with a similar study by Ghosh et al. which mentioned that most patients in their study wanted to continue chemotherapy despite the pandemic. ${ }^{[10]}$

Only a small percentage of patients (84/864, 9.72\%) underwent a change in their treatment plan. Most of these treatment plans were modified by the treating physician with a view to minimize patients' exposure to the hospital system without deviating from standard treatment guidelines.

Eleven bone marrow transplants were carried out without inflicting any additional complications on these immunocompromised patients.

Twenty-one deaths $(21 / 864,2.43 \%)$ were reported in our study. This was comparable to the number of deaths $(26 / 1157,2.24 \%)$ observed in a similar cohort of patients treated in our hospital in the 2 months of January and February 2020. Only two deaths were due to COVID infection in patients with advanced hematological malignancies.

Despite the presence of 363 comorbidity factors in 864 patients, there was no increased susceptibility to infection.

During the study period, the number of COVID cases in the city, state, and our hospital was rising exponentially [Figures $1 \mathrm{~b}-\mathrm{c}$ and 6].

Despite more than 5500 essential hospital visits in 864 patients during this period, there was minimal incidence of COVID infection in the study patients.

This model of effective patient segregation, separation of treatment streams, and judicious use of testing has resulted in minimal treatment delays or changes, is unlikely to have an adverse impact on the outcome of cancer, and can be effectively employed in a resource-limited setting in light of the pandemic. Possibly, the actual risk of treating cancer patients in the COVID pandemic in a COVID hospital may be much less that what has been estimated. A similar sentiment has been echoed in the correspondence by Cai et al., with regard to mortality in COVID-positive patients undergoing surgery. ${ }^{[1]]}$

\section{Conclusion}

We successfully delivered cancer treatment to patients in our DCH. Only $12.61 \%$ of our patients reported a delay in treatment initiation due to the pandemic situation. We modified the treatment plan for $8.72 \%$. The percentage of adverse effects, symptomatic COVID infection, and related mortality has been very low in our study. Thus, we would like to conclude that cancer care - a semi-emergency in itself, can be continued with due diligence even during this pandemic.

\section{Strength of the study}

We have reported our experience of treating cancer patients in a hospital which was simultaneously managing COVID 


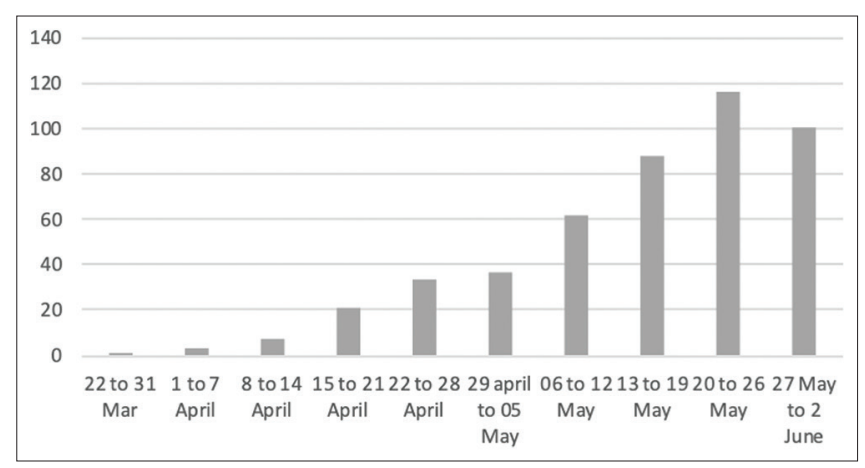

Figure 6: Weekly admissions of COVID-19 patients in our hospital during the study period

patients in an area which was a COVID hotspot. To the best of our knowledge, such a combined study including surgery, chemotherapy, and radiotherapy has not been reported in the recent COVID-related literature.

We continued to manage cancer patients in a COVID hospital, without any major compromise in a pandemic situation, especially when the scientific and medical community was leaning toward postponement and change in the treatment of most cancers. ${ }^{[1,12]}$ This speaks a lot about the preparedness of the hospital team as a whole, and it can be attributed to diligent training, motivation, and setting up of SOPs for each service.

\section{Limitations}

It can be argued that most of these patients have not been tested for COVID infection unless they have had COVID-like symptoms or exposure to contacts. The clinical significance of these asymptomatic undiagnosed carriers is not properly known. In this pandemic, it is difficult to generate randomized data. In addition, there is no similar historical precedent for comparison.

The effective management of COVID and cancer patients under a single roof was possible because the graph of COVID patients in Pune and India is still on an upward curve and the health-care systems are not yet overwhelmed with cases. Upcoming months may present different challenges.

\section{Generalizability}

Cancer treatment cannot be postponed indefinitely due to the fear of disease progression and worsening of outcomes. A judicious balance of continuing cancer treatment along with management of the pandemic needs to be considered in a resource-limited country like India, where tertiary care hospitals will have to take the initiative.

\section{Acknowledgment}

The authors wish to acknowledge the contribution of the following collaborators with regard to data collection and analysis.
Dr. Meetkumar Trivedi, Dr. Sunanda Harwalkar, Dr. Jyoti Mohite, Dr. Vidyadevi Manjare, Dr. Ashish Kulkarni, Dr. Pradnya Kore, Dr. Manjusha Sutar, Mrs. Vrushali, and Mrs. Suneeta.

\section{Financial support and sponsorship}

Nil.

\section{Conflicts of interest}

There are no conflicts of interest.

\section{References}

1. Krishnamurthy, A., Gopinath, K.S. The need for prioritizing cancer surgeries amidst the COVID-19 pandemic. Indian J Surg Oncol 2020;11:169-70.

2. Lei S, Jiang F, Su W, Chen C, Chen J, Mei W, et al. Clinical characteristics and outcomes of patients undergoing surgeries during the incubation period of COVID-19 infection. EClinicalMedicine 2020;21:100331.

3. Advisory for Hospitals and Medical Institutions dated $20^{\text {th }}$ March 2020. Ministry of Health and Family Welfare. Government of India. Available from: https://www.mohfw.gov.in. [Last accessed on 2020 Jun 30].

4. Pramesh CS, Badwe RA. Cancer management in India during COVID-19. N Engl J Med 2020;382:e61.

5. Jog S, Kelkar D, Bhat M, Patwardhan S, Godavarthy P, Dhundi U, et al. Preparedness of acute care facility and a hospital for COVID-19 pandemic: What we did! Indian J Crit Care Med 2020;24:385-92. Available from: https://www.ijccm. org/doi/IJCCM/pdf/10.5005/jp-journals-10071-23416. [Last accessed on 2020 Jul 02].

6. Cancer Patient Management during the COVID-19 Pandemic. Available from: https://Esmo.org/guidelines. [Last accessed o 2020 Jun 30].

7. ICMR.GOV.IN COVID-19 Testing Strategy. Revised Strategy of COVID-19 Testing in India (Version 3) 20/03/2020. Available from: https://ICMR.GOV.IN. [Last accessed on 2020 Jul 02].

8. Nahshon C, Bitterman A, Haddad R, Hazzan D, Lavie O. Hazardous postoperative outcomes of unexpected COVID-19 infected patients: A call for global consideration of sampling all asymptomatic patients before surgical treatment. World J Surg 2020;44:2477-81.

9. Shrikhande SV, Pai PS, Bhandare MS, Bakshi G, Chaukar DA, Chaturvedi $\mathrm{P}$, et al. Outcomes of elective major cancer surgery during COVID-19 at Tata Memorial Centre: Implications for cancer care policy. Ann Surg 2020;272:e249-e252.

10. Ghosh J, Ganguly S, Mondal D, Pandey P, Dabkara D, Biswas B. Perspective of oncology patients during COVID-19 pandemic: A prospective observational study from India. JCO Glob Oncol 2020;6:844-51.

11. Cai M, Wang G, Zhang L, Gao J, Xia Z, Zhang P, et al. Performing abdominal surgery during the COVID-19 epidemic in Wuhan, China: A single-centred, retrospective, observational study. Br J Surg 2020;107:e183-5.

12. Bartlett DL, Howe JR, Chang G, Crago A, Hogg M, Karakousis G, et al. Management of cancer surgery cases during the COVID-19 pandemic: Considerations. Ann Surg Oncol 2020;27:1717-20. 